\title{
Comparison among different therapeutic techniques to treat low back pain: a monitored randomized study
}

\author{
Luca Morelli, ${ }^{1,2}$ Simona Carla Bramani, ${ }^{1}$ Marco Cantaluppi,, ${ }^{2}$ Mara Pauletto, ${ }^{2}$ Alessandro Scuotto ${ }^{2}$ \\ 'San Fedele Physiotherapy Clinic, Longone al Segrino (CO), Italy; ${ }^{2}$ LUdeS Foundation Higher \\ Education Institution, Lugano, Switzerland
}

\begin{abstract}
Idiopathic low back pain can be considered as a chronic condition, characterized by recurrent episodes of pain and functional limitation. The aim of this study is to compare two therapeutic methods to treat this chronic disease: the oxygen-ozone therapy and the diathermy through Tecar ${ }^{\circledR}$ therapy. Two groups of 10 patients each who suffered from postural idiopathic low back pain due to different pathologies have been recruited. All selected patients have been evaluated through spinometry and have been given the Oswestry low back pain disability questionnaire to fill in at the beginning of the treatments and at the end of them with a three-month follow-up. The first group underwent a diathermy treatment through Tecar ${ }^{\circledR}$ therapy, whilst the second group received an oxygen-ozone therapy treatment through a paravertebral lumbar infiltration; both treatments have been associated with a standard physiokinesitherapy treatment. Data collected through Formetric spinometry show an improvement in both groups, but in the second group (treated with oxygen-ozone therapy+physiokinesitherapy), the improvement is greater (from $6 \%$ to $57 \%$ ) against the first group (from $20 \%$ to $38 \%$ ). In conclusion, the study has cor roborated the validity of both treatments leading to improvement of symptomatology, but while one treatment leads to some relapses after a few months, the second one has a greater healing effect, which preserves over time.
\end{abstract}

Correspondence: Luca Morelli, San Fedele Physiotherapy Clinic, via Risorgimento 1, 22030 Longone al Segrino (CO), Italy.

Tel.: +39.031.3333585.

E-mail: info@centrosanfedele.it

Key words: Idiopathic low back pain; oxygen-ozone therapy; diathermy; Tecar $^{\circledR}$ therapy; physiokinesitherapy.

Received for publication: 12 January 2016.

Accepted for publication: 24 February 2016.

(C) Copyright L. Morelli et al., 2016

Licensee PAGEPress, Italy

Ozone Therapy 2016; 1:5842

doi:10.4081/ozone.2016.5842

This article is distributed under the terms of the Creative Commons Attribution Noncommercial License (by-nc 4.0) which permits any noncommercial use, distribution, and reproduction in any medium, provided the original author(s) and source are credited.

\section{Introduction}

\section{Definition}

Idiopathic low back pain can be considered to all intents and purposes, as a chronic condition, characterized by recurrent episodes of pain and functional limitation. Low back pain may have different origins, since any pathology of the lumbar spine can cause pain in this region. However, the most common causes are the degenerative ones that is to say due to the wear and tear of disc and articular structures. Degenerative forms shall be differentiated from those due to other causes.

\section{Low back pain classification}

\section{Discogenic}

Its occurrence is observed mainly in the age group 30 to 50 . It is caused by the degenerative discopathy of one or more intravertebral discs. The most affected discs are L4-L5 and L5-S1. The low back pain can be acute, chronic or recurrent.

\section{Arthrogenic}

Its occurrence is observed in patients over 50 . Usually the low back pain is chronic, however in most cases it is less intense than the discogenic one. The instrumental examinations [X-rays (XR), computed tomography (CT), nuclear magnetic resonance (NMR)] show arthrosic alterations of back articulations at low lumbar spine level or in the spine in general and often also the narrowness of one or more discs due to degenerative alterations.

Due to vertebral instability

The pain is only present during the prolonged upright standing, the protracted walking, or during physical efforts. In these cases it is usually a degenerative spondylolisthesis, noticeable through the lateral XR, with hypermobility of the concerned vertebra in flexionextension XR.

Due to other causes

The pain in the lumbar region, which is usually chronic, can be caused by several other vertebral pathologies, such as: tumors, infections o rheumatic diseases, extra-vertebral pathologies (retroperitoneal tumors, abdominal aortic aneurysm).

In the majority of cases in which no pathological alterations to lumbar spine are found (through the standard XR, NMR methods), the low back pain is idiopathic, that is to say, without any known cause.

It usually concerns young or middle-aged people, whose pain can be caused by postural defects during their working activity, by an inade- 
quate mobilization of the spine or by the manual handling of loads, which is performed incorrectly.

In many cases in idiopathic low back pain there is a pain psychogenic component, above all in hypochondriac or depressed subjects.

\section{Materials and Methods}

\section{The study}

We have planned this study to compare two therapeutic methods to treat idiopathic low back pain: the oxygen-ozone therapy and the diathermy through Tecar $^{\circledR}$ therapy, associated with a standard Physiokinesitherapy treatment.

\section{Type of patients}

We have recruited two groups made up of 10 patients each who suffered from postural idiopathic low back pain due to pathologies caused by lumbosacral intradiscal conflicts such as: disc bulging, lumbar protrusion and discophaties.

\section{Inclusion criteria}

We have considered those patients with a medical diagnosis stating disc bulging, protrusion or intradiscal conflict documented by CT or NMR; patients aged between 25 and 70; the onset of symptoms not previous to 12 months.

\section{Exclusion criteria}

We have excluded from the study those subjects suffering from systemic diseases (rheumatic diseases, osteoporosis, etc.); evident hernias; hernia recurrence; vertebral fractures; pseudosciatica.

\section{Recruiting method}

We have used the controlled random method. The names of those patients showing homogeneous inclusion characteristics have been put into individual anonymous envelops and then divided by the treatment to examine by a third person who was unaware of the study under consideration.

We evaluated 25 patients and then selected 20 of them, since 4 were suffering from extruded hernia and 1 from pseudosciatica with piriformis muscle syndrome, therefore according to the exclusion criteria they could not be admitted.

\section{Work execution}

\section{Evaluation}

Both groups have been evaluated before and after treatment by means of Diers - Formetric 4D of the company Hako-Med Italia sas [Egna (BZ), Italy], an instrument for the posture spinometric test and the analysis of spine mechanical pathologies. The Formetric analysis system uses the video rastereography, an innovative and very accurate method determined by the possibility to re-create, through a dedicated software, the real tridimensional structure of the spine and to define automatically a spatial ratio between dorsal morphology and bony skeleton.

Thanks to the Formetric vertebral morphology analysis system it is no longer necessary to have recourse to the manual application of markers since it automatically detects the reference marks, the median line of the spine and its rotation.

Spinometry represents the result of the examination showing the tridimensional model of the spine, with a representation of its morphology and rotation with the addition of the pelvis position.

All patients selected according to the above-mentioned inclusion and exclusion criteria, have been evaluated through spinometry and have been given the Oswestry low back pain disability questionnaire to fill in at the beginning of the treatments and at the end of them with a threemonth follow-up.

\section{The Oswestry low back pain disability questionnaire}

It consists in a percentage assessment scale of disabilities during the daily activities caused by low back pain. The patient fills in a questionnaire divided into 10 sections made up of 5 multiple-choice answers aimed at assessing: i) pain intensity; ii) personal care (to wash hands and face, brush teeth, comb, shave and put on make-up, etc.); iii) manual activities; iv) walking; v) staying seated; vi) standing; vii) sleeping; viii) sexual activity; ix) public relations; $\mathrm{x}$ ) moving and travelling.

Once the questionnaire has been filled up, the answers of the individual sections are added up to obtain the final score which, therefore, can have a maximum of 50 scores. Such value is then multiplied by two in order to get a percentage value corresponding to the disability percentage.

\section{The Formetric spinometry}

It determines the tridimensional reconstruction of the spine by using the triangulation and video rasterstereography principles without emitting radiations.

According to the results of spinometric test, the ten data obtained by each group after a comparison between the first and the last finding with Formetric are evaluated, then it is performed a follow-up after three months. The ten variables of the Formetric analysis taken into consideration are: i) pelvic torsion; ii) righ surface rotation; iii) left surface rotation; iv) maximum kyphotic angulation; v) maximum lordotic angulation; vi) pelvic inclination; vii) right side deviation; viii) left side deviation; ix) cervical curve; $x$ ) lumbar curve.

\section{Method of analysis}

We have processed data concerning the 0swestry percentages and calculated their standard means and deviations, by availing ourselves of the Student T statistic test to analyze the significance of differences among the individual means.

In our analysis we made reference to $\mathrm{T}$ values by 18 degrees of freedom, using as threshold value of P-value the standard 0.05 . In this case $\mathrm{T}$ shall always be greater than 1.734 in order to consider the difference among the means as significant (Table 1).

Instead, data obtained by Formetric findings during the first and the last session, have been analyzed in both groups by comparing the improvement, invariance and deterioration percentages of single postural components indicated by the spinometric test.

\section{Comparison between treatment protocols}

\section{First group}

To the patients of the first group, who have been selected with the methods mentioned above, has been applied a diathermy treatment through Tecar $^{\circledR}$ therapy associated with a standard physio-kinesitherapeutic treatment.

\section{Second group}

The second group's patients were administered an oxygen-ozone therapy treatment through a paravertebral lumbar infiltration associated with a standard physio-kinesitherapeutic treatment.

\section{Diathermy through Tecar ${ }^{\circledR}$ therapy}

Patients underwent 10 Pharon diathermy sessions by the company Mectronic [Mectronic Medicale Srl, Grassobbio (BG), Italy] through 
Tecar $^{\circledR}$ therapy and physiokinesitherapy twice a week, for five weeks totally.

For the Tecar $^{\circledR}$ therapy treatment we have used a stainless-steel receiving plate with some conductive cream for diathermy from Medisport applied on the patient's abdominal region when laying in prone position, on patient's lumbar region we applied the same conductive cream to facilitate the transmitting electrode sliding.

Patients have been treated in capacitive mode for $8 \mathrm{~min}$ to an intensity that allowed the patient to feel a warm sensation in the treated area, we went on for other $8 \mathrm{~min}$ in resistive mode while trying again to obtain the same sensation on the patient and finally one last 8minute session in capacitive mode.

\section{Oxygen-ozone therapy}

According to the oxygen-ozone therapy, we decided to follow the therapeutic protocol mentioned here below entailing paravertebral injections.

Applications have been administered once a week for the first three weeks by decreasing the dose once every two weeks.

The oxygen-ozone infiltration has been performed in symmetric areas of those paravertebral muscle masses near the hernia localization, with a concentration of $18-20 \mu \mathrm{g} / \mathrm{mL}$ of ozone and a quantity equal to 5 cc per site of inoculation, for a total of 4 paravertebral infiltrations per session and of 6 sessions with infiltrations once a week for the first $3 / 4$ weeks, and then every 2 weeks.

For the administration of oxygen-ozone we used Multiossigen srl [Gorle (BG), Italy] machineries, in particular the Medical 99 IR and Medical 98HCPS.

\section{Results}

In Figures 1 and 2 we report the charts showing the results obtained by the Oswestry's questionnaire administration.

By analyzing data collected by means of the Oswestry low back pain disability questionnaire, it can be noticed that the improvement occurred from the first to the last session for the two groups is similar. Instead, the difference between the two results of follow-up changes

Table 1. Standard T distribution.

\begin{tabular}{rrrrrr}
\multicolumn{1}{r}{$v$} & \multicolumn{5}{c}{$\alpha$} \\
1 & 0.10 & 0.050 & 0.025 & 0.010 & 0.005 \\
2 & 3.078 & 6.314 & 12.706 & 31.821 & 63.657 \\
3 & 1.886 & 2.920 & 4.303 & 6.965 & 9.925 \\
4 & 1.638 & 2.353 & 3.182 & 4.541 & 5.841 \\
5 & 1.533 & 2.132 & 2.776 & 3.747 & 4.604 \\
6 & 1.476 & 2.015 & 2.571 & 3.365 & 4.032 \\
7 & 1.440 & 1.943 & 2.447 & 3.143 & 3.707 \\
8 & 1.415 & 1.895 & 2.365 & 2.998 & 3.499 \\
9 & 1.397 & 1.860 & 2.306 & 2.896 & 3.355 \\
10 & 1.383 & 1.833 & 2.262 & 2.821 & 3.250 \\
11 & 1.372 & 1.812 & 2.228 & 2.764 & 3.169 \\
12 & 1.363 & 1.796 & 2.201 & 2.718 & 3.106 \\
13 & 1.356 & 1.782 & 2.179 & 2.681 & 3.055 \\
14 & 1.350 & 1.771 & 2.160 & 2.650 & 3.012 \\
15 & 1.345 & 1.761 & 2.145 & 2.624 & 2.977 \\
16 & 1.341 & 1.753 & 2.131 & 2.602 & 2.947 \\
17 & 1.337 & 1.746 & 2.120 & 2.583 & 2.921 \\
18 & 1.333 & 1.740 & 2.110 & 2.567 & 2.898 \\
19 & 1.330 & 1.734 & 2.101 & 2.552 & 2.878 \\
20 & 1.328 & 1.729 & 2.093 & 2.539 & 2.861 \\
& 1.325 & 1.725 & 2.086 & 2.528 & 2.845
\end{tabular}

significantly (Figure 3): while the first group recorded $22.80 \%$ of disability, the second one remained at $12.40 \%$, with a noticeable percentage gap equal to $10.40 \%$. This means that the second group maintained the obtained result over time with the treatment sessions.

Analyzing the data collected through the analysis with Formetric spinometry it is possible to notice an improvement in both groups under examination (Figures 4 and 5).

\section{\% Oswestry comparison First group}

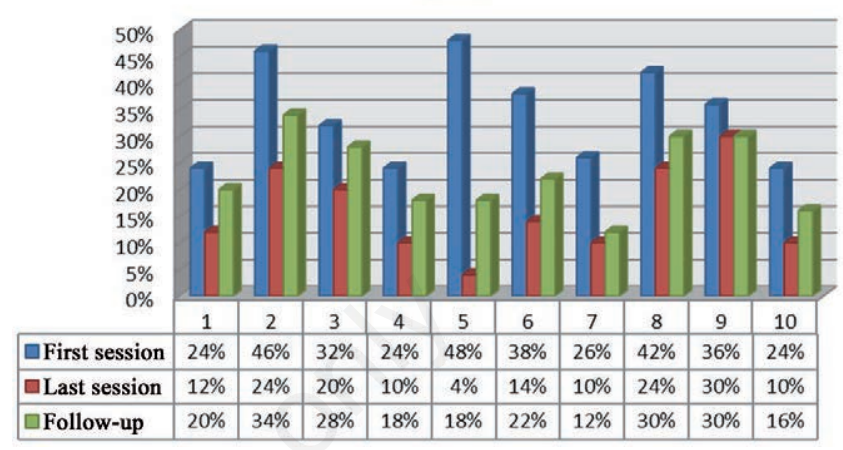

Figure 1. Percentage comparison, first group's assessment scale.

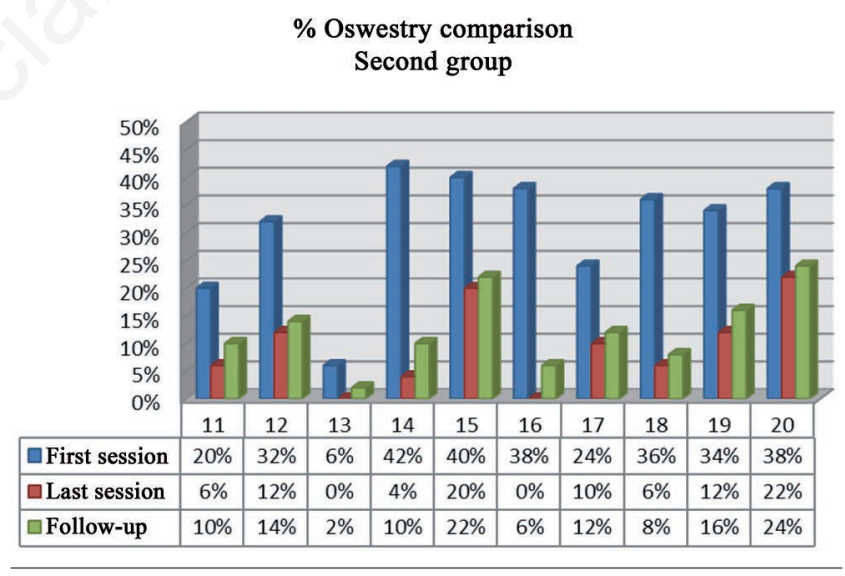

Figure 2. Percentage comparison, second group's assessment scale.

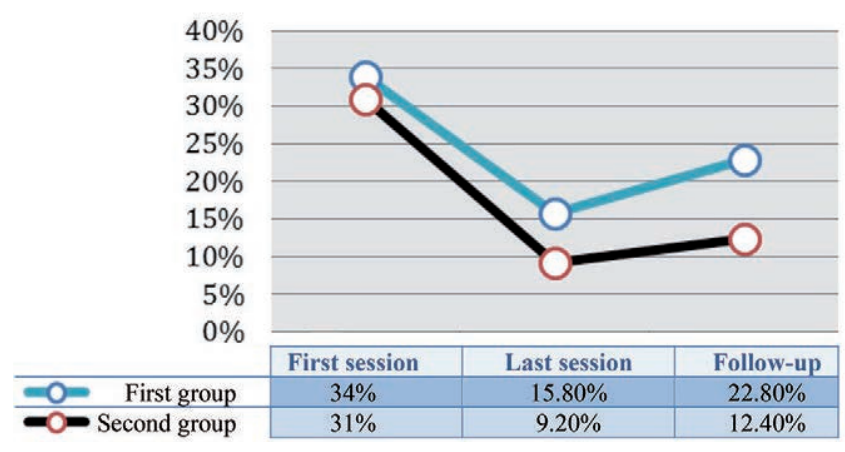

Figure 3. Comparison between the two results of follow-up. 
Comparison among findings with formetric $-1^{\text {st }}$ group

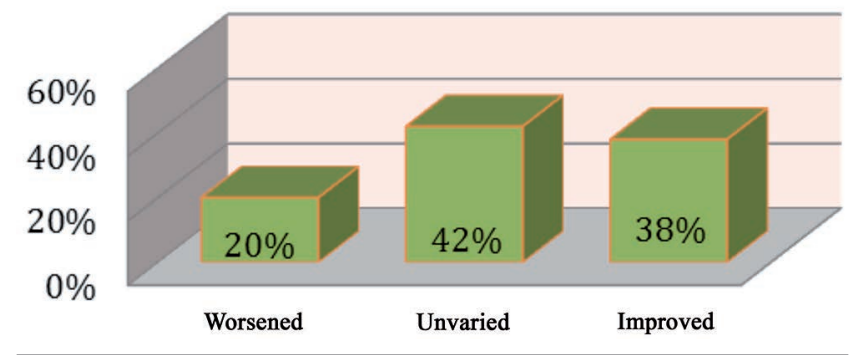

Figure 4. Comparison of first group's results.
The improvement is greater in the second group, the one treated with oxygen-ozone therapy associated with standard physiokinesitherapy, for which the improvement data increase from $6 \%$ to $57 \%$, against those of the first group increasing from $20 \%$ to $38 \%$.

\section{Discussion and Conclusions}

Data analyzed at the end of the two types of treatment and through follow-up after three months showed the following results.

Through the Oswestry low back pain disability questionnaire we can observe how patients belonging to the first group, the one that underwent the therapy with diathermy through Tecar $^{\circledR}$ therapy, have all reported an improvement immediately after the therapeutic cycle.

This has been also confirmed by the spinometric tests performed, from which it is possible to see that the majority of detected parameters showed a real improvement in all patients, emphasized by the progressive normalization, towards the normality range for the majority of data detected.

A similar situation can be observed for the second group treated with oxygen-ozone therapy.

By analyzing the answers to the questionnaire the improvement has been grater compared to the first one. Data pointed out with Formetric show a marked improvement.

According to the comparison between the two therapeutic methods used, from the results obtained by the questionnaire filling in and the Formetric findings, we can observe the following:

- Patients treated with diathermy through Tecar ${ }^{\circledR}$ therapy achieved a general improvement of $18.2 \%$ from the first to the last session and a slight worsening comparable to $7 \%$ from the last session to the follow-up performed after three months.

- Patients treated with oxygen-ozone therapy recorded a greater improvement, equal to $21.8 \%$, both from the first to the last session and during the revaluation after three months, showing a worsening of only $3.2 \%$.

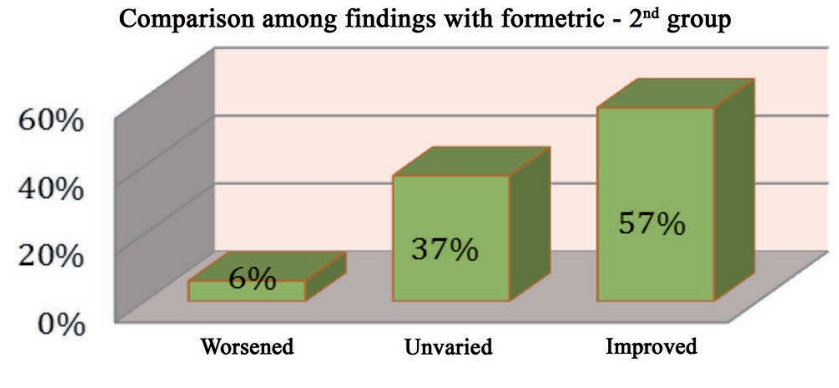

Figure 5. Comparison of second group's results.

Therefore we can assert that both methods produced positive results for the majority of patients suffering from idiopathic low back pain, however those who underwent diathermy show a greater worsening upon follow-up compared to those who were treated with oxygen-ozone therapy who preserve the beneficial effect of treatments for a longer time.

We can conclude by saying that the work has corroborated the validity of both treatments that led to both a subjective and objective improvement of symptomatology.

While one treatment leads to some relapses after a few months, the second one has a greater healing effect, which preserves over time.

\section{References}

1. Mangone M. La Spinometria Formetric nella valutazione non invasiva delle deformità del rachide e del tronco. Congress on Biomedical Products, La Sapienza University for HAKOMED Italia, 2010 May 8-9, Rome, Italy.

2. Kapandji IA. Fisiologia articolare. Vol. 3: Tronco e rachide. Milano: Monduzzi Ed.; 1994.

3. Romano D. Spinometria, nuova frontiera per diagnosi e terapie. Giorn Previdenza Med Odontoiatr 2008;10:24-5. Available from: https:/www.enpam.it/wp-content/uploads/10_2008.pdf

4. Benizet MP, Colmer JF. Tecarterapia nella patologia del ginocchio e della colonna vertebrale; 2003. Available from: http:/www.cmconsulenze.it/pdf/tecar_evidenze_cliniche/patologia_ginocchio_colon na_vertebrale.pdf

5. Chiefari M, Verde N, Salerno N, Luongo C. Efficacia antalgica dell'ozonoterapia. $6^{\text {th }}$ SIO0T National Congress 1994, Rome, Italy.

6. Bertè F, Vairetti M, Richelmi P. Ozono: problemi tossicologici con particolare riguardo alla formazione di radicali liberi'. $2^{\text {nd }}$ SIOOT National Congress 1990, Punta Ala (GR), Italy. 